\title{
Infection Control Precautions and Care Delivery in Hemodialysis Unit during Coronavirus Disease 2019 Outbreak: A Case Series
}

\author{
Shou-Ci Hu ${ }^{a}$ En-Qing Fu ${ }^{a}$ Jun-Fen Fan ${ }^{a}$ Qing-Qing $\mathrm{Ye}^{\mathrm{a}}$ Cong Xia \\ Tie-Er Gan ${ }^{\mathrm{b}}$ Hong-Zhen Ma ${ }^{\mathrm{a}}$ \\ aDepartment of Nephrology, The First Affiliated Hospital of Zhejiang Chinese Medical University (Zhejiang \\ Provincial Hospital of Traditional Chinese Medicine), Hangzhou, China; ${ }^{b}$ Department of Hospital Infection, The \\ First Affiliated Hospital of Zhejiang Chinese Medical University (Zhejiang Provincial Hospital of Traditional Chinese \\ Medicine), Hangzhou, China
}

\section{Keywords}

Coronavirus disease $2019 \cdot$ Hemodialysis · Dialysis unit · Infection control precautions · Care delivery

\begin{abstract}
Background: With an estimated basic reproductive number of 3.77, the Coronavirus Disease 2019 (COVID-19) continues to spread. It is urgent to exert adequate efforts for the management of dialysis patients, caregivers, and healthcare personnel (HCP). This study aimed at reporting practical workflow, identification of high-risk or suspected cases of COVID-19, and subsequent response measures. Methods: At the time of the COVID-19 outbreak, precautions and practice protocols were applied in our dialysis units (DUs). This singlecenter study retrospectively reviewed all high-risk/suspected cases from January 23, 2020, to February 10, 2020. Epidemiological, clinical feature, and detailed data on all cases were recorded. Results: Practical workflow for the clinical management of dialysis patients, caregivers, and HCP was initiated. A total of 6 high-risk/suspected cases were identified. Female gender, older age, presence of cardiovascular disease, diabetes, anuresis, immunocompromised status, hypoalbuminemia, and underweight were noticeable features in these cases. Direct evidence of infection or epide-
\end{abstract}

miological risk was detected in five cases. Close monitoring for temperature and oxygen saturation during hemodialysis sessions may be reasonable. No confirmed COVID-19 cases were reported in our DU, but certain cases showed rapid deterioration due to other critically severe condition needing hospitalization. Portable dialysis machines are of great need to ensure dialysis care provision. Conclusions: Our study described a practical workflow for patient-centered management during COVID-19 outbreak. Potential risk factors and underlying clinical patterns were reported. Further studies regarding the efficacy of infection control precautions and practice protocols tailored for dialysis settings are warranted.

๑) 2020 S. Karger AG, Basel

\section{Introduction}

It is the third time since the early 21 st century that a zoonotic coronavirus (SARS-CoV-2) has crossed species to bring about serious public health events. In December 2019 , initial cases with exposure to a wet market that developed acute respiratory disease were first reported in Wuhan, China [1]. Due to its alarming scale of spread and severity, the World Health Organization (WHO) de-

$\begin{aligned} & \text { karger@karger.com } \\ & \text { www.karger.com/bpu }\end{aligned}$
Karger ${ }^{\prime /}$


clared the current coronavirus disease (COVID-19) a global pandemic [2]. Like the previous outbreaks caused by coronaviruses, such as the severe acute respiratory syndrome (SARS) and Middle East respiratory syndrome (MERS) coronavirus, COVID-19 tends to cause unfavorable outcomes. By March 14, 2020, a total of 118,319 cases have been confirmed globally, affecting 135 countries worldwide, and a total of 4,292 deaths have been reported throughout the world [3].

Tracing back to 2003, a medical ward outbreak of SARS associated with 4 dialysis patients was reported in Hong Kong [4]. During the last epidemic, a dialysis unit (DU) outbreak of MERS was reported in Saudi Arabia in 2013 [5]. The uremia state, immunocompromised status, comorbidity burden, frequent hospital visit, and the extracorporeal nature of the therapy are potential risk factors making hemodialysis (HD) patients prone to severe infections. Furthermore, dialysis care delivery requires close contact and multi-person attendance; therefore, DU is at high risk during virus outbreaks. So far, it is unclear how many dialysis patients are involved in the COVID-19 pandemic. A recent study from Wuhan showed that the main causes of death among dialysis patients with $\mathrm{CO}$ VID-19 were cardiovascular/cerebrovascular disease and hyperkalemia, which was mainly due to the reduction of dialysis times since patients were in fear of contracting the virus [6]. In light of the current pandemic, it is important to exert adequate effort for the management of dialysis patients, caregivers, and healthcare personnel (HCP). The aim of this study is to report practical workflow regarding the infection control precautions and dialysis care delivery, description of high-risk/suspected cases of COVID-19, and subsequent response measures with a case series from our HD units.

\section{Materials and Methods}

Infection control guidance and practice suggestions have been updated several times by the national workgroups (i.e., new coronavirus pneumonia national prevention and control program) to cope with the changing situation. Given the SARS experience, dialysis patients may present with less typical clinical characteristics [7]; thus, the criteria for a suspected case in dialysis settings need to be adjusted based on the guidance from the WHO [8] and national workgroups [9] with a combination of dialysis expertise, relaxing the definition of a suspected case or a case of high risk when appropriate. At the time of the COVID-19 outbreak, precautions and clinical practice protocols were adopted and applied in our DU in January 2020. The workflow is shown in Figure 1. As a part of the hospital infection prevention and control (IPC) protocol, the north wing of the inpatient building was set up for isolation use, isolation wards with an intensive care unit team were preserved for critical care, and doctors and nurses with dialysis expertise have joined the workforce. Elevators and transportation routes for segregation and cohorting were designated upon patients' arrival.

COVID-19 testing is mandatory for all patients who will require vascular access procedures. Interdisciplinary consultation and coordination were planned for non-dialysis-dependent patients who developed fever and respiratory illness while being in need of urgent start of dialysis. Rapid turnaround testing and screening for these patients were prioritized. The detailed flowchart is illustrated in Figure 2.

For screening purpose, laboratory testing for complete blood count, high-sensitivity C-reactive protein, serum biochemistry, identification of other respiratory pathogens (such as influenza A virus [H1N1, H3N2, H7N9], influenza B virus, respiratory syncytial virus, and adenovirus), and computed tomography of the thorax were applied to all high-risk cases. Following the isolation precautions, outpatients were sent to the fever clinic, which was located at the entrance of the hospital and was separated from the main entrance, while inpatients were sent to a separate room in the isolation ward. Contact and droplet precautions, including N95 respirator, gloves, waterproof disposable gowns, and eye protection, were applied to HCP and patients, when appropriate.

During January 23, 2020-February 10, 2020, a total of 129 patients of end-stage kidney disease (ESKD), ranging in age from 32 to 90 years, received HD sessions at the DU of the First Affiliated Hospital of Zhejiang Chinese Medical University, Hangzhou, China (geographical location is $30^{\circ} 16^{\prime}$ North, $120^{\circ} 12^{\prime}$ East, and the city has a registered population of 9.806 million in 2018). Among these, 6 patients presented with mild to moderate signs of infection and were identified as suspected or high-risk cases.

The data were obtained from the blood purification center of the First Affiliated Hospital of Zhejiang Chinese Medical University. Informed consent was signed before HD sessions. All suspected cases were systematically reviewed for exposure history where the local epidemiological situation was adapted, clinical features (new-onset symptoms and signs, laboratory results, and thorax CT scans), demographics, cause of ESKD, comorbidity, dialysis vintage, residual kidney function, presence of potential risk factors (diabetes and immunosuppressive therapy), Barthel [10] index for activities of daily living, admission history, clinical courses, and patient outcomes. Virology laboratory procedures were performed in accordance with the WHO protocol [11] in the laboratory unit of the First Affiliated Hospital of Zhejiang Chinese Medical University, and nasal or pharyngeal swab specimens were collected for high-throughput sequencing or real-time RT-PCR assay in all suspected or high-risk cases. A laboratory-confirmed case with COVID-19 was defined as a positive result to highthroughput sequencing or RT-PCR assay for nasopharyngeal and oropharyngeal swabs [12], according to the WHO interim guidance [8].

\section{Results}

As of February 10, 2020, a total of 6 patients, ranging in age from 30 to 80 years, with a median vintage of 23.5 (interquartile range 3.75-42.50) months were identified as high-risk or suspected cases. Among these, 4 patients 


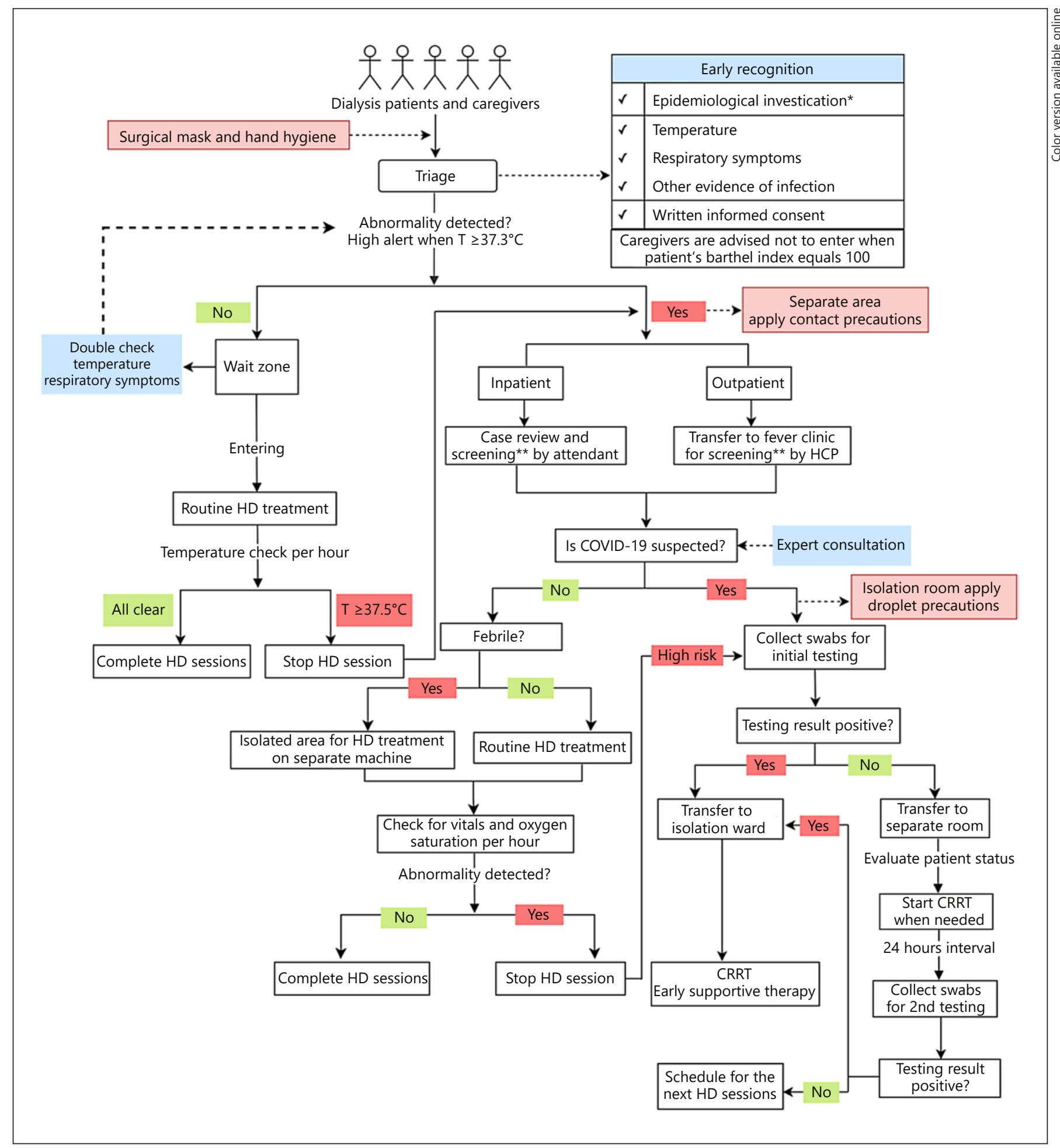

Fig. 1. Workflow for patient-centered management in a dialysis setting during COVID-19 outbreak. *Epidemiological investigation includes travel history, contact history, and other exposure history where the local epidemiological situation was adapted, as described in the WHO interim guidance [8]. ${ }^{* *}$ Screening includes clinical assessment, laboratory testing for complete blood count, high-sensi- tivity C-reactive protein, serum biochemistry, identification of other respiratory pathogens (such as influenza A virus [H1N1, H3N2, H7N9], influenza B virus, respiratory syncytial virus, and adenovirus), and computed tomography of the thorax. HD, hemodialysis; HCP, healthcare personnel; COVID-19, coronavirus disease 2019; CRRT, continuous renal replacement therapy. 


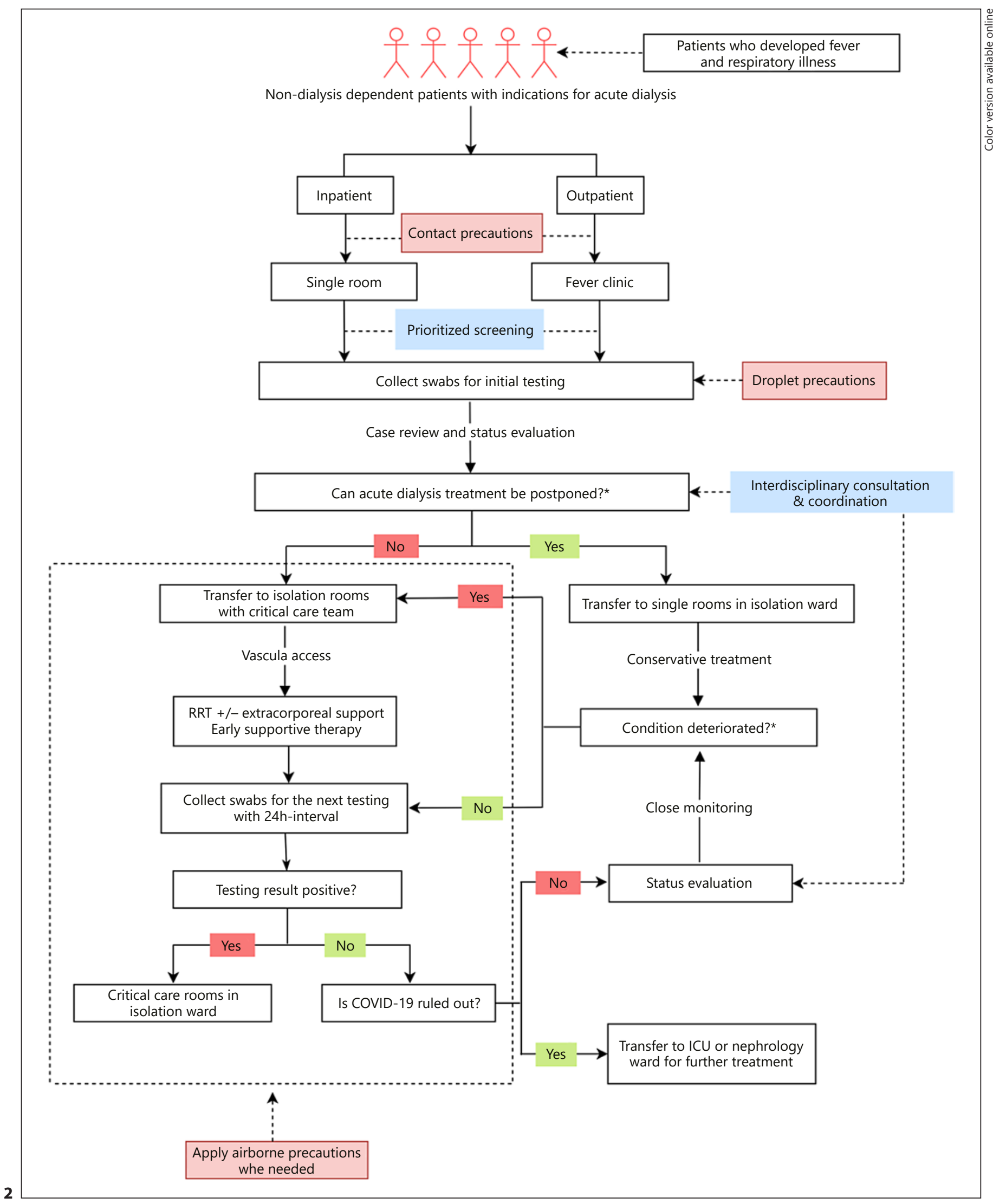

(For legend see next page.) 
were female (4/6), 3 patients were diabetic (3/6), 4 patients had comorbid cardiovascular disease (4/6), 3 patients were anuretic (3/6), and one patient from the inpatient setting was on glucocorticoids for the treatment of idiopathic thrombocytopenic purpura (1/6). Lower level of serum albumin $(\leq 38 \mathrm{~g} / \mathrm{L})$ and low BMI $\left(\leq 18.5 \mathrm{~kg} / \mathrm{m}^{2}\right)$ were reported in 2 cases. The baseline demographic and clinical features are shown in Table 1.

No patient had a travel history to Hubei Province, and 1 patient had an exposure history within the last 14 days to a local source of transmission. Respiratory symptoms

Table 1. Baseline demographic and clinical features for patients with suspected/high-risk of COVID-19

\begin{tabular}{lc}
\hline Variable & $\begin{array}{c}\text { Value }(n=6) \text { median } \\
\text { (IQR) or count/no. of } \\
\text { patients, } \%\end{array}$ \\
\hline Age, years & $65(59-72)$ \\
Age $>60$ years & $4 / 6(66.67 \%)$ \\
Female gender & $4 / 6(66.67 \%)$ \\
HD vintage, months & $23.50(3.75-42.50)$ \\
Primary cause of ESKD & \\
Glomerulonephritis & $2 / 6(33.33 \%)$ \\
Diabetic kidney disease & $2 / 6(33.33 \%)$ \\
Unknown & $2 / 6(33.33 \%)$ \\
Diabetes & $3 / 6(50 \%)$ \\
Cardiovascular disease & $4 / 6(66.67 \%)$ \\
Hypertension & $6 / 6(100 \%)$ \\
Cancer & $1 / 6(16.67 \%)$ \\
Under immunosuppressive therapy & $1 / 6(16.67 \%)$ \\
BMI, kg/m ${ }^{2}$ & $19.43(17.68-20.58)$ \\
Urine output, mL/24 h & $150(0-1200)$ \\
Hemoglobin, g/L & $105(94-110)$ \\
Serum albumin, g/L & $39.5(38.0-41.0)$ \\
Phosphorus, mmol/L & $1.86(1.56-2.14)$ \\
From inpatient settings & $1 / 6(16.7 \%)$ \\
Barthel index $(0-100)<100$ & $1 / 6(16.67 \%)$ \\
Three HD sessions per week & $6 / 6(100 \%)$ \\
\hline &
\end{tabular}

COVID-19, coronavirus disease 2019; IQR, interquartile range; $\mathrm{HD}$, hemodialysis; ESKD, end-stage kidney disease.

Fig. 2. Workflow for the management of non-dialysis-dependent patients who have indications for acute dialysis when COVID-19 is suspected. *Referring to immediate or delayed dialysis [26]; the latter means to start dialysis in response to severe metabolic acidosis, severe hyperkalemia, pulmonary edema, $72 \mathrm{~h}$ of oliguria, or a blood urea concentration of $>37 \mathrm{mmol} / \mathrm{L}$. RRT, renal replacement therapy; ICU, intensive care unit; COVID-19, coronavirus disease 2019.

Precautions and Care Delivery in Hemodialysis Unit during COVID-19 and low oxygen saturation level were reported in 2 cases. Of the 5 cases with symptoms of fever, 2 were spotted at triage and 3 were detected during HD sessions. Detailed information on 6 suspected/high-risk cases is shown in Table 2.

After the screening procedure, evidence of infection such as abnormal total blood cell count, elevated level of high-sensitivity C-reactive protein or procalcitonin, and radiographic findings related to pneumonia was found in 3 cases. Criticallevel of potassium $(6.9 \mathrm{mmol} / \mathrm{L})$ was reported in 1 case and partial pressure of oxygen dropping was found in 2 cases. After evaluation, 2 patients were transferred to an isolation/separate room in the ICU for bedside continuous renal replacement therapy (CRRT), and 1 patient was admitted to the isolation ward due to exposure history and respiratory symptoms.

Nasopharyngeal swab specimens were collected for virology tests, and two consecutive negative results (at least $24 \mathrm{~h}$ apart) were reported in all cases. Two cases with deteriorating condition requiring hospitalization were transferred to separate rooms in the nephrology ward for further treatment. After 2 days of monitoring, the remaining 4 patients were afebrile and no deterioration or new-onset symptoms were reported.

All 6 patients except for 1 patient without direct evidence of infection or epidemiological risk were scheduled in an isolated area on separate machines for their next HD sessions.

\section{Discussion/Conclusion}

With an estimated basic reproductive number varing in range from 2.7 to 4.2 [13], COVID-19 continues to spread. Due to the complexity of dialysis patients' clinical picture, early implementation of IPC protocols in the DU is of paramount importance. In this study, we have reported a practical workflow for IPC and care delivery in a dialysis setting. Given different DU layout and design standards, it is impossible to unify routes in all dialysis settings. However, planning ahead for a worst-case scenario is advisable, such as necessary structural alteration, room partitioning for contingency use, contact surveillance, appropriate patient allocation strategies, intensified equipment cleaning and disinfection policies, and response measures in the event of detection of a suspected or confirmed case. It was reported that over $90 \%$ of the confirmed MERS-CoV cases in a DU were acquired by person-to-person transmission. During Ebola outbreak, 
Table 2. Detailed information on 6 suspected/high-risk cases of COVID-19

\begin{tabular}{|c|c|c|}
\hline Case detail & $\begin{array}{l}\text { Value (median [IQR]) } \\
\text { or count/patients, } n(\%)\end{array}$ & Extra information \\
\hline Epidemiological risk & $1 / 6(16.67)$ & $\begin{array}{l}\text { Patient has travel history within the last } 14 \text { days to a local endemic } \\
\text { city }\end{array}$ \\
\hline Temperature $\geq 37.5^{\circ} \mathrm{C}$ & $5 / 6(83.33)$ & Median (min-max) range of temperature: $37.7(37.1-39.2)^{\circ} \mathrm{C}$ \\
\hline Febrile detected at triage & $2 / 6(33.33)$ & \\
\hline Oxygen saturation $<93 \%$ on room air & $2 / 6(33.33)$ & \\
\hline Mean arterial pressure, $\mathrm{mm} \mathrm{Hg}$ & $115(105-128)$ & \\
\hline White blood cell count, $\times 10^{9} / \mathrm{L}$ & $5.7(5.0-6.5)$ & \\
\hline Neutrophil percentage, $\%$ & $72.45(63.40-80.15)$ & \\
\hline Abnormal ABGs & $2 / 6(33.33 \%)$ & Arterial oxygen pressure dropping $\left(\mathrm{PaO}_{2} 63.6-71.7 \mathrm{~mm} \mathrm{Hg}\right)$ \\
\hline Hyperkalemia & $1 / 6(16.67)$ & Critical level $(K=6.5 \mathrm{mmol} / \mathrm{L})$ \\
\hline \multirow[t]{4}{*}{ Radiographic findings in chest CT scan } & \multirow[t]{4}{*}{$4 / 6(66.67 \%)$} & $\begin{array}{l}\text { Patient 1: coarse patchy shadowing and peribronchial nodules in the } \\
\text { upper lobe of the left lung }\end{array}$ \\
\hline & & $\begin{array}{l}\text { Patient 2: solid nodules and focal fibrosis in the lower lobe of the left } \\
\text { lung }\end{array}$ \\
\hline & & $\begin{array}{l}\text { Patient 3: peripheral increased opacity with scattered fibrous stripes } \\
\text { in the lower lobe of the right lung }\end{array}$ \\
\hline & & $\begin{array}{l}\text { Patient 4: focal consolidation with peripheral increased opacity in } \\
\text { the lower lobe of the left lung, bilateral pleural effusion with lower } \\
\text { lobe atelectasis }\end{array}$ \\
\hline Need hospitalization & $2 / 6(33.33 \%)$ & \\
\hline Afebrile after 2 days of monitoring & $4 / 6(66.67 \%)$ & \\
\hline Rescheduled in an isolated area for the next HD sessions & $5 / 6(83.33 \%)$ & \\
\hline
\end{tabular}

COVID-19, coronavirus disease 2019; IQR, interquartile range; hs-CRP, high-sensitivity C-reactive protein; ABGs, arterial blood gas test; ICU, intensive care unit; CRRT, continuous renal replacement therapy; SARS-CoV-2, severe acute respiratory syndrome coronavirus 2; HD, hemodialysis.

Fresenius Medical Care (FMC) activated multidisciplinary collaboration [14]; measures including daily situation assessment through teleconference, providing staffassisted HD limiting the exposed patients at home, evaluating exposure risk among HCP, preserving space for isolation, and applying contact precautions when needed substantially improved the capacity and preparedness for
IPC and care delivery in dialysis settings during the epidemic.

Epidemiological studies showed that older age, diabetes, hypertension, and cardiovascular disease were risk factors among COVID-19 patients, indicating worse outcomes [15]. In this study, we have described cases with high risk or suspected infection of SARS-CoV-2. More 
than half of the identified cases were females, aged over 60 years, and had comorbid cardiovascular disease. Furthermore, half of the patients involved were diabetic and anuretic. Presence of immunocompromised status, hypoalbuminemia, and underweight were also noticeable in these cases.

Another particularly important lesson we learned from previous virus outbreaks is early identification of highrisk and suspected cases. As it was discussed during SARS outbreaks, dialysis patients tend to have atypical presentations, which is difficult to distinguish from uremic symptoms. Fever should set a high alert at the point of entry; however, only one-third of the febrile cases were detected at triage in our study. Close monitoring for temperature and oxygen saturation during HD sessions may be reasonable. On the other hand, more attention should be paid to differential diagnosis and timely evaluation. Although case confirmation mostly depends on virological testing, CT features of viral pneumonia may be strongly suspicious for COVID-19 despite negative RT-PCR results [16]. In our study, laboratory testing for complete blood count, hs-CRP, serum biochemistry, identification of other respiratory pathogens, and computed tomography of the thorax were included during the screening phase to help validate differential diagnosis. So far no confirmed COVID-19 cases were reported in our DU, but certain cases showed rapid deterioration due to other critically severe conditions. Since it is no longer a safe option to admit such patients in the nephrology ward for in-center dialysis, portable dialysis machines or CRRT machines are in great need to ensure dialysis care provision. Finally, after patients have been temporarily ruled out of $\mathrm{CO}$ VID-19, appropriate allocation and isolation strategies should be taken into account when rescheduling for the next HD sessions.

Based on the successful implementation of the renal replacement therapy, the last decade has witnessed a great advance in blood purification techniques. As the frontline of multiple organ support, extracorporeal organ support [17], including VV-ECMO, ECCO2R, VA$\mathrm{ECMO}$, intermittent and continuous HD, hemofiltration, hemodiafiltration, hemoperfusion, plasmapheresis, and sorbent therapies, has served its role in life support and provided an opportunity to improve patient outcomes in critical care settings [18-20]. It was reported that $5 \%$ of the confirmed cases was in need of intensive care during the COVID-19 outbreak [21]. Renal impairment was found in $63 \%$ of the COVID-19 cases in one ongoing case study [22], which may contribute to multiple organ failure and death [23]. As the situation of the current pandemic evolves, extracorporeal organ support readiness has become a crucial part of care delivery [24]. The golden hour of the rescue window in treatment of endotoxic shock was discussed before in the literature [25]. Despite the uncertainty of ideal timing, acute assessment in high-risk patients is fundamental; thus, rapid screening and turnaround testing for patients in need of acute dialysis are prioritized in our setting. In the context of COVID-19, awareness has been raised that ECMO itself may trigger the escalation of cytokine storm, and the combination of CRRT and hemoperfusion may be the solution to reduce cytokine insult [21]. Given the potential increase in renal replacement therapy utilization and anticipated ethical dilemmas, a paradigm shift is inevitable; organized and practical IPC measures in both dialysis and intensive care settings will go a long way in the current pandemic.

It is our unshakable duty to ensure dialysis care delivery in this emergency period. To our knowledge, clinical studies regarding IPC measures and clinical management of COVID-19 tailored to meet the needs in dialysis settings are scarce. Our study may serve the purpose of providing practical workflow for patient-centered management, and potential clinical feature identification of highrisk cases during the ongoing pandemic.

\section{Acknowledgements}

We would like to thank all the hard-working doctors and nurses in our blood purification center for their excellent medical care and assistance in data collection. All authors had access to the data and a role in writing the manuscript.

\section{Statement of Ethics}

This research was conducted ethically in accordance with the World Medical Association Declaration of Helsinki. Patients enrolled in our study have given their written informed consent, and the study protocol was approved by the Ethics Committee of the First Affiliated Hospital of Zhejiang Chinese Medical University.

\section{Disclosure Statement}

The authors have no conflicts of interest to declare.

\section{Funding Sources}

This work was supported by Zhejiang Natural Science Foundation (Grant No. LGF18H050003). 


\section{Author Contributions}

The specific work of each author in this study was as follows: Shou-Ci Hu and En-Qing Fu: conception and design, data interpretation, writing (including draft, review, and editing), and finalization of the manuscript. Jun-Fen Fan: data validation, supervi- sion, and manuscript review. Qing-Qing Ye and Cong Xia: data acquisition, data collection, and data analysis. Tie-Er Gan: data validation and supervision. Hong-Zhen Ma: conceptualization, resources, supervision, article drafting, and final approval of the version to be published.

\section{References}

1 Li Q, Guan X, Wu P, Wang X, Zhou L, Tong $\mathrm{Y}$, et al. Early transmission dynamics in $\mathrm{Wu}-$ han, China, of novel coronavirus-infected pneumonia.NEnglJMed.2020;382(13):1199207.

2 World Health Organization. Novel coronavirus (2019-NCoV) situation report-51. 2020. Available from: https://www.who.int/docs/ default-source/coronaviruse/situationreports/20200311-sitrep-51-covid-19.pdf.

3 World Health Organization. Coronavirus disease 2019 (COVID-19) situation report-54. 2020. Available from: https://www. who.int/docs/default-source/coronaviruse/ situation-reports/20200314-sitrep-54-covid-19.pdf.

4 Wong PN, Mak SK, Lo KY, Tong GM, Wong $\mathrm{Y}$, Watt CL, et al. Clinical presentation and outcome of severe acute respiratory syndrome in dialysis patients. Am J Kidney Dis. 2003;42(5):1075-81.

5 Assiri A, McGeer A, Perl TM, Price CS, Al Rabeeah AA, Cummings DA, et al. Hospital outbreak of middle east respiratory syndrome coronavirus. N Engl J Med. 2013;369(5):40716.

6 Ma Y-Q, Diao B, Lv X-F, Zhu J-L, Liang W, Liu L, et al. 2019 novel coronavirus disease in hemodialysis (HD) patients: report from one HD center in Wuhan, China. 2019 novel coronavirus disease in hemodialysis (HD) patients: report from one HD center in Wuhan, China. medRxiv. 2020. https://doi.org/10.110 1/2020.02.24.20027201.

7 Kwan BC, Leung CB, Szeto CC, Wong VW, Cheng YL, Yu AW, et al. Severe acute respiratory syndrome in dialysis patients. J Am Soc Nephrol. 2004;15(7):1883-8.

8 World Health Organization. Clinical management of severe acute respiratory infection when novel coronavirus (2019-nCoV) infection is suspected: interim guidance. 2020.
Available from: https://www.who.int/publications-detail/clinical-management-of-severe-acute-respiratory-infection-when-novel-coronavirus-(ncov)-infection-is-suspected.

9 New coronavirus pneumonia prevention and control program (2nd ed.). 2020. Available from: http://www.nhc.gov.cn/jkj/s3577/ $202001 /$ c67 cfe 29 ecf 1470 e 8 c7fc 47 d 3b751e88.shtml.

10 Mahoney FI, Barthel DW. Functional evaluation: the barthel index. Md State Med J. 1965; 14. $61-5$.

11 World Health Organization. Laboratory testing for 2019 novel coronavirus (2019-nCoV) in suspected human cases. 2020. Available from: https://www.who.int/health-topics/coronavirus/laboratory-diagnostics-for-novelcoronavirus.

12 Chen N, Zhou M, Dong X, Qu J, Gong F, Han $\mathrm{Y}$, et al. Epidemiological and clinical characteristics of 99 cases of 2019 novel coronavirus pneumonia in Wuhan, China: a descriptive study. Lancet. 2020;395(10223):507-13.

13 Zhu Y-F, Chen Y-Q. On a Statistical Transmission Model in Analysis of the Early Phase of COVID-19 Outbreak. Stat Biosci. 2020. https://doi.org/10.1007/s12561-020-09277-0. In press.

14 Boyce JM, Hymes JL. What we learned from ebola: preparing dialysis units for the next outbreak. Clin J Am Soc Nephrol. 2018; 13(4):669-70.

15 Guan W-J, Ni Z-Y, Hu Y, Liang W-H, Ou C-Q, He J-X, et al., China Medical Treatment Expert Group for Covid-19. Clinical Characteristics of Coronavirus Disease 2019 in China. N Engl J Med. 2020;382(18):1708-20.

16 Xie X, Zhong Z, Zhao W, Zheng C, Wang F, Liu J. Chest CT for typical 2019-nCoV pneumonia: relationship to negative RT-PCR testing. Radiology. 2020;12:200343.
17 Ronco C, Ricci Z, Husain-syed F. From multiple organ support therapy to extracorporeal organ support in critically Ill patients. Blood Purif. 2019;48(2):99-105.

18 De Rosa S, Samoni S, Ronco C. Sequential extracorporeal therapy collaborative device and timely support for endotoxic, septic, and cardiac shock: a case report. Blood Purif. 2019. https://doi.org/10.1159/000505146. In press.

19 Ferrari F, Carletti A, Peroni N, Mongodi S, Esposito P, Orlando A, et al. Brief report: a case of tramadol overdose: extracorporeal life support and hemoperfusion as life-saving treatment. Blood Purif. 2020. https://doi. org/10.1159/000505845. In press.

20 Ankawi G, Xie Y, Yang B, Xie Y, Xie P, Ronco C. What have we learned about the use of cytosorb adsorption columns? Blood Purif. 2019;48(3):196-202.

21 Ronco C, Reis T, De Rosa S. Coronavirus epidemic and extracorporeal therapies in intensive care: si vis pacem para bellum. Blood $\mathrm{Pu}$ rif. 2020;49(3):255-8.

22 Li Z, Wu M, Yao J-W, Guo J, Liao X, Song S-J, et al. Anti-2019-nCoV Volunteers. Caution on Kidney Dysfunctions of COVID-19 Patients. medRxiv. 2020. https://doi.org/10.110 1/2020.02.08.20021212.

23 Husain-Syed F, Rosner MH, Ronco C. Distant organ dysfunction in acute kidney injury. Acta Physiol. 2020;228:e13357.

24 Ronco C, Navalesi P, Vincent J. Coronavirus epidemic: preparing for extracorporeal organ support in intensive care. Lancet Respir Med. 2020;8(3):240-1. .

25 Rosa SDe, Villa G, Ronco C. The golden hour of polymyxin $\mathrm{B}$ hemoperfusion in endotoxic shock : the basis for sequential extracorporeal therapy in sepsis. Artif Organs. 2020;44(2): 184-6.

26 Bellomo R, Kellum JA, Ronco C. Acute kidney injury. Lancet. 2019;380(9843):756-66. 